\title{
Flow Anlaysis on Hal Tejas Aircraft using Computational Fluid Dynamics with Different Angle of Attack
}

\author{
Abhishek Jain ${ }^{1}$, S. K. Sharma ${ }^{2}$, Comandur Rajasekhar Kaushik ${ }^{3}$, Abhishek Gour ${ }^{4}$ \\ ${ }^{1,2}$ Assistant Professor \\ ${ }^{1,2,3,4}$ Mechanical and Automation Engineering, Amity University Rajasthan, Jaipur, India
}

\begin{abstract}
In the current globalization, we can see many innovations being introduced or implemented in every aspect of field that are considered to be existed. Every country is aiming to develop its power over all the aspects that considered for comparison with other countries in order to stand at same level of competition with others. One such power considered by all countries to develop every possible way to have a healthy competition is the military power which involves basically innovations of fast moving aircraft having a high lift coefficient and low drag coefficient. Such an aircraft having the high lift and low drag coefficient is TEJAS (HAL) developed by country India on which the purpose of paper mainly sustains. The paper mainly focuses on steady-state flow analysis over aircraft TEJAS using the computer aided modelling techniques and also the comparison of the results obtained from the modelled techniques. The paper also outlines the designing of the structural model of the TEJAS in a modelling software, creation of a finite computational domain, segmentation of this domain into discrete intervals, applying boundary conditions such as velocity in order to obtain plots and desired results determining the coefficient of pressure, lift and drag coefficient, velocity magnitude etc. This paper also aims in creating awareness to the future students about the techniques involved and knowledge required for developing a designed modelled. This paper also highlights the use of CFD techniques involved for the purpose of fluid flow simulation of the aircraft especially performing the meshing techniques, pre and post processing techniques and finally the evaluation of the simulation. Finally this paper can be seen as source by future generation students in gaining knowledge about design, analysis and simulation of the structured model on various conditions, about the field of aerospace engineering and new innovations being developed and also about the career
\end{abstract}

involved when the above fields were chosen foe specialization purposes

Keywords-Ansys Fluent, Catia Software.

\section{INTRODUCTION}

An aircraft is a well designed structured body basically used for moving in air by gaining the strength from atmosphere as it moves against the force of gravity. In earlier days aircraft were seen as transportation medium but with the increase in new ideas and innovations, aircraft were introduced into the military power, thus we could see aircraft flying during the world wars. As development in the globalization continued, the development in many fields also enhanced. With these developments we can see many new innovations in designs of aircraft to make it as innovative as possible. One such innovation were, the considerations of properties such as the static and dynamic lift to counter the gravitational force in order to fly the aircraft at great heights and also to withstand against the force of thrust. Thus the present generation aircrafts include a lot of multidisciplinary designs to achieve critical mission requirements such as endurances, manoeuvrability, fuel consumption, payload capacity, noise emission etc when compared to the older ones. But these critical requirements can only be achieved with the help of aerodynamic property of an aircraft such as the drag, vortex, etc. The aerodynamic force is found to be a central idea behind the design of an aircraft. An important aspect of this aerodynamic force is the induced drag that approximates about $33 \%$ of the total aerodynamic force when caused by the lift and during the low speed movement achieved at the time of landing and takeoff situation, it accounts for $80 \%-90 \%$ of the total drag. The streamline present on an aircraft is also important aspect of design in determining difference in pressure between upper and lower surface of the aircraft body. The finite wings present in an aircraft also plays major role in 
designing since at the tip of the wing, there is leakage of air molecules. So these finite wings are provided with the wing tip sails in order to produce the required thrust during the generation of lift. However research are still performed in order to bring new innovations in these designs of aircraft body and also to increase its efficiency. Apart from these characteristics some of the other important aspects considered for an aircraft design are the environmental factors such as types of pollution, sudden climatic changes due to increase in global warming and many other factors that play a vital role for future growth. Nowadays travelling in air depends mostly on the environment thus making the power factor in the aircraft design. The figure below shows the structured model of an aircraft.

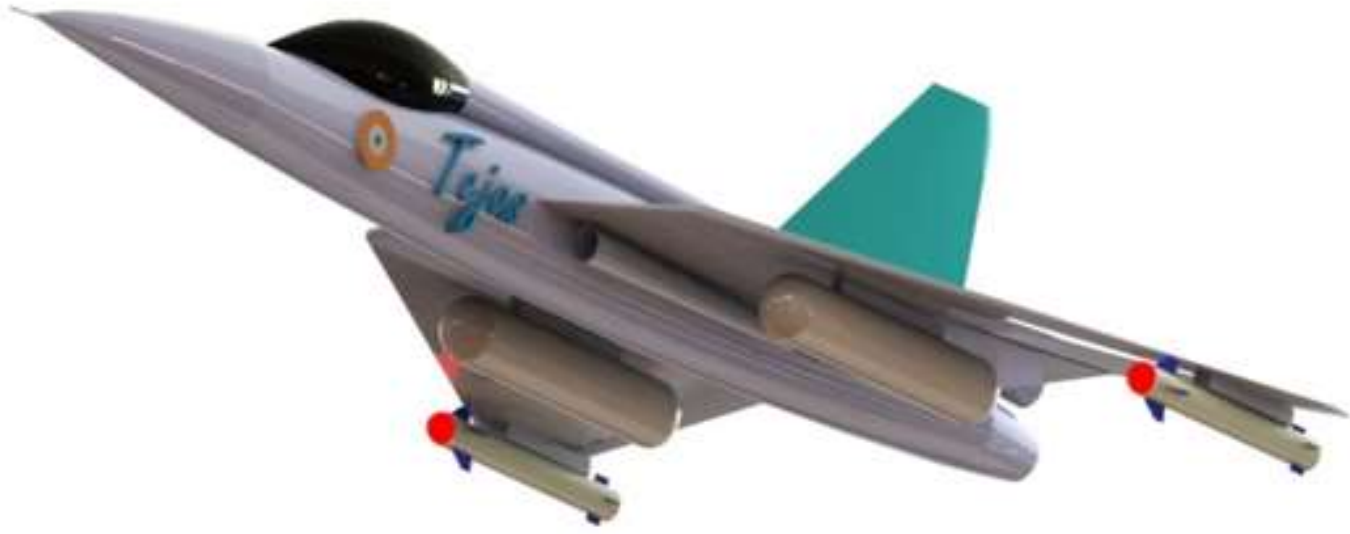

Fig.1: Modeling of F-16 HAL TEJAS Aircraft using Solid works 2015

The structured model shown above is designed from the specifications of the model TEJAS introduced by Hindustan Aeronautical Limited, India. The aircraft TEJAS is well developed product which came into light from the Light Combat Aircraft (LCA) programme in the year 1980. It was considered as replacement to the single fighter flights since TEJAS is a multi-role fighter. The design of TEJAS consists of many disciplinarians out of which some properties such as the highly supersonic, highly manoeuvrable and also the lightest body are considered important. It is made of composite material structures and the aerodynamic design, is a combination of an intense design process involving wind tunnel studies and utilization of extensive Computational Fluid Dynamics, the subject which basically used for the simulation of structured models for the analysis of the fluid flow. It has Specific aerodynamic features that provide excellent aircraft performance in a wider flight envelope. The dimensions of TEJAS considered during the design of structured model are, Wing span which is about $8.20 \mathrm{~m}$, while length and height measures about $13.20 \mathrm{~m}$ and $4.40 \mathrm{~m}$. The weight of the aircraft while takeoff clean is $9800 \mathrm{~kg}$, during empty is $6560 \mathrm{~kg}$ and about external stores is $3500 \mathrm{~kg}$. Its max speed is supersonic at all altitudes. Its service ceiling is $50,000 \mathrm{ft}$ and ' $g$ ' limits are $+8 /-3.5$. $^{(12)}$. Apart from the consideration of these specifications for the design of the structured model, the study about the area Computational Fluid Dynamics (CFD) was also done for the further proceeding of the paper.CFD is a branch of study of fluid mechanics involving algorithms and numerical methods to solve and analyse the problems of fluid flows. Nowadays CFD is basically studied for performing advanced calculation purposes that are achieved by running the software. With the introduction this software, any complex scenarios involving turbulent flows or transonic flows can be calculated with accuracy and speed. Many of the flight tests involving validation of the properties considered during the design and simulation analysis of the structural body of design on the basis of fluid flow can be achieved easily with this CFD based software. The calculation of Navier-Stokes equations is easily achieved through this software that is basically used for calculating the single phase fluid flows. Apart from these terms another term considered in this paper is the "Angle of Attack", on which the whole results of the paper are been drawn. It is basically defined as the angle between a reference line on a body (often the chord line of an airfoil) and the vector representing the relative motion between the body and the fluid through which it is moving. The below figure the structured design of the model TEJAS be shown in terms of measurement of "Angle of Attack". 


\section{ANGLE OF ATTACK}

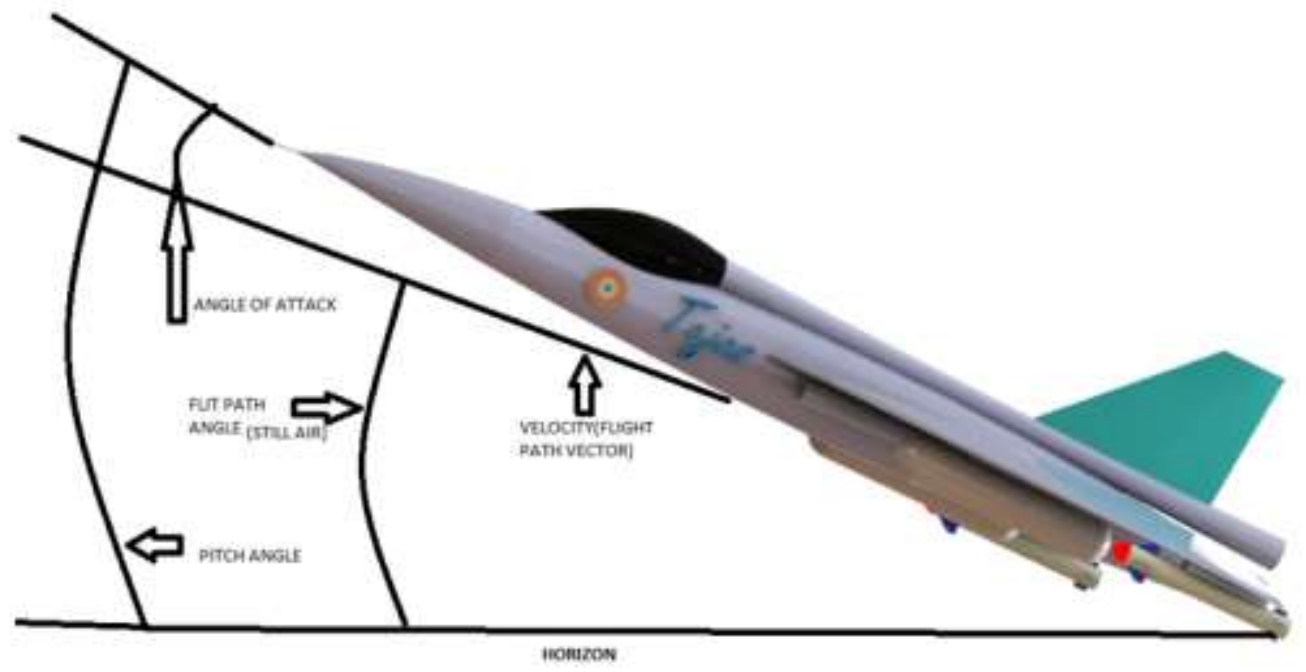

Fig.2: Showing the basic measure of Angle of Attack

\section{METHODOLOGY AND BOUNDARY CONDITIONS}

\section{A. Creation of geometry:}

The structural model of TEJAS is designed using the SOLID WORKS 2015 software for the purpose of simulation and analysis. ${ }^{(11)}$

\section{B. Mesh generation:}

The designed model was imported into ANSYS Work Bench and meshed appropriately. The meshing tools like mapping and inflation cannot be employed while meshing as it is symmetrical in shape and gives a very fine mesh. Once the process of meshing is completed, it is then exported to ANSYS Fluent 14, where the simulation of fluid flow analysis is performed. ${ }^{(11)}$

\section{Simulation of the Continuum:}

Here the simulation of structured body is performed in ANSYS Fluent 14. In this the meshing of the body is checked and once the software approves it, the models, materials and boundary conditions are set. ${ }^{(11)}$

\section{Model:}

The model used for this kind of simulation is the K-E $\operatorname{model}^{(11)}$

\section{Materials:}

The air is considered as a working fluid for the purpose of simulation in this paper and is allowed to act on the aircraft at an altitude of 50,000 ft. Only density is considered as the material property of the air and is constant i.e. $1.225 \mathrm{~kg} / \mathrm{m} 3$. ${ }^{(11)}$

\section{Boundary Conditions:}

The important boundary conditions considered for the purpose of the external flow analysis are the Mach number which is considered as inner boundary condition, is taken as Mach2 i.e. the velocity of inlet taken as $553 \mathrm{~m} / \mathrm{s}$ and speed of sound at this altitude is $334.72 \mathrm{~m} / \mathrm{s}$. The outer boundary condition is taken as pressure and its value taken as 0 Pascal. The rest of the faces of continuum are considered symmetry which means that the faces are under no-slip condition i.e. flow condition does not apply on these faces. ${ }^{11}$

4. Solution:

Once the boundary conditions are set, the solution methods and controls are set for this simulation. The solution method set for this is the coupled solver. And as for the solution controls the courant number is set to 0.25 and the under relaxation factors for momentum and pressure are set as 0.75 and for the turbulent kinetic energy, turbulent dissipation rate and turbulent viscosity is set to $0.8 .^{(11)}$

5. Analysis:

The CFD Analysis and study of results are carried out in 3 steps: (1) Pre-processing, (2) Solving and (3) Post-processing by using fluent solver in ANSYS work bench. $\left({ }^{11)}\right.$

Velocity Inlet: The inlet boundary conditions involve velocity components for varying angle of attack, turbulence intensity and turbulent viscosity ratio. ${ }^{(11)}$

Pressure Outlet: Ambient atmospheric condition is imposed at outlet. The velocity components are calculated for each angle attack case as follows. The $\mathrm{x}$-component of 
velocity is calculated by $\mathrm{x}=\mathrm{u} \cos (\mathrm{x})$ and the $\mathrm{y}$ component of velocity is calculated by $\mathrm{y}=\mathrm{u} \sin (\mathrm{x})$, where $(\mathrm{x})$ is the angle of attack in degrees. $\left({ }^{11)}\right.$

Symmetry: This is the boundary wall to see the analysis. ${ }^{(11)}$ Operating condition: The inlet velocity taken for fuselage $533 \mathrm{~m} / \mathrm{sec}$, for different angle of attack of air at normal degree, 4 degree and 8degreee and at STP (Temperature $=300 \mathrm{k}$, Pressure $=101325 \mathrm{pa}$ ) as the fluid medium. ${ }^{(11)}$

\section{Properties of Air: ${ }^{(11)}$}

Density: $\rho=1.225 \mathrm{~kg} / \mathrm{m} 3$

Viscosity: $\mu=1.7894 \mathrm{e}-05 \mathrm{~kg} / \mathrm{m}-\mathrm{s}$

Solvers selection: Pressure based solvers.

Mathematical models: K- $\boldsymbol{\varepsilon}$ standard wall function

Solution controls: Gauss-Seidel flow turbulence energy.

Momentum: Second Order Upwind Scheme

Initialization: Inlet Values

Force Monitors: Lift and Drag

Reference values: Inlet Values

Convergence Limit: $1 \times 10^{-9}$

\section{RESULTS AND DISCUSSION}

The analysis to be performed on all cases is carried out in ANSYS FLUENT software. Results of the analysis are drawn and are shown below using Analysis Post processor. Velocity, pressure and residual plots are plotted on all cases of study.

\section{Test case I:}
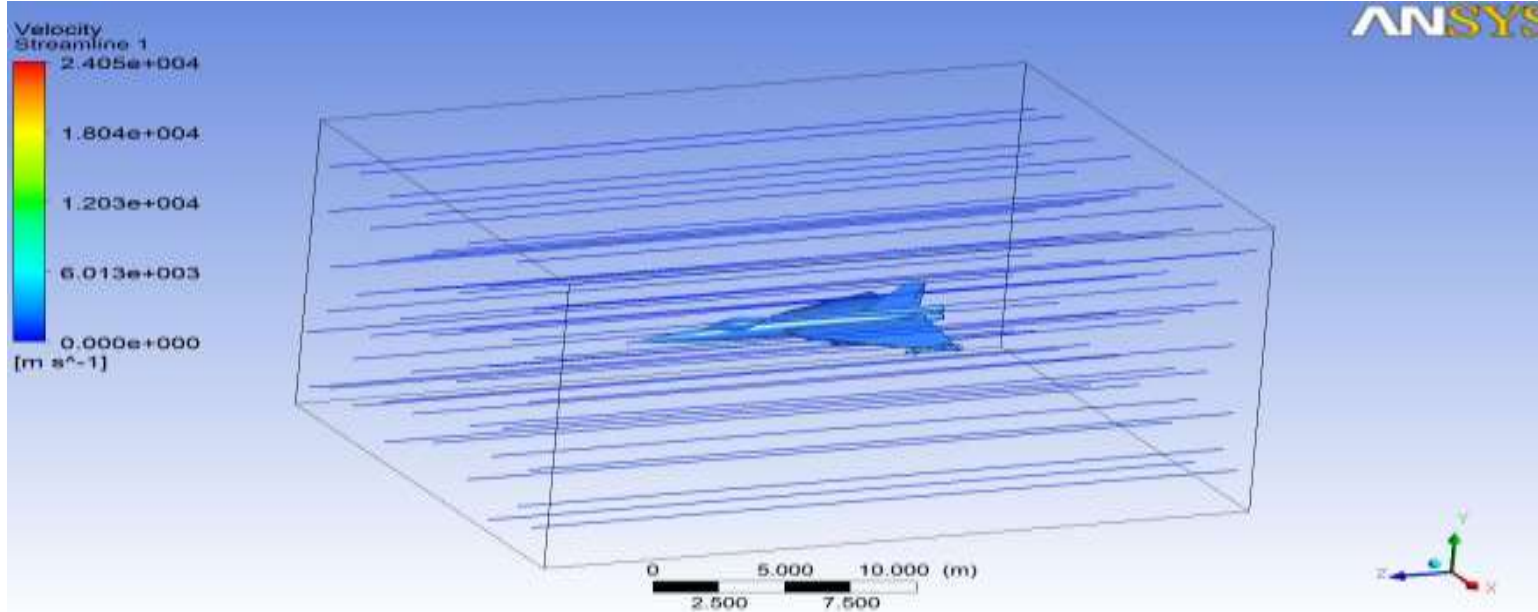
$\left[m s^{n-1}\right.$

Fig.3.1: Velocity contour at a normal degree angle .

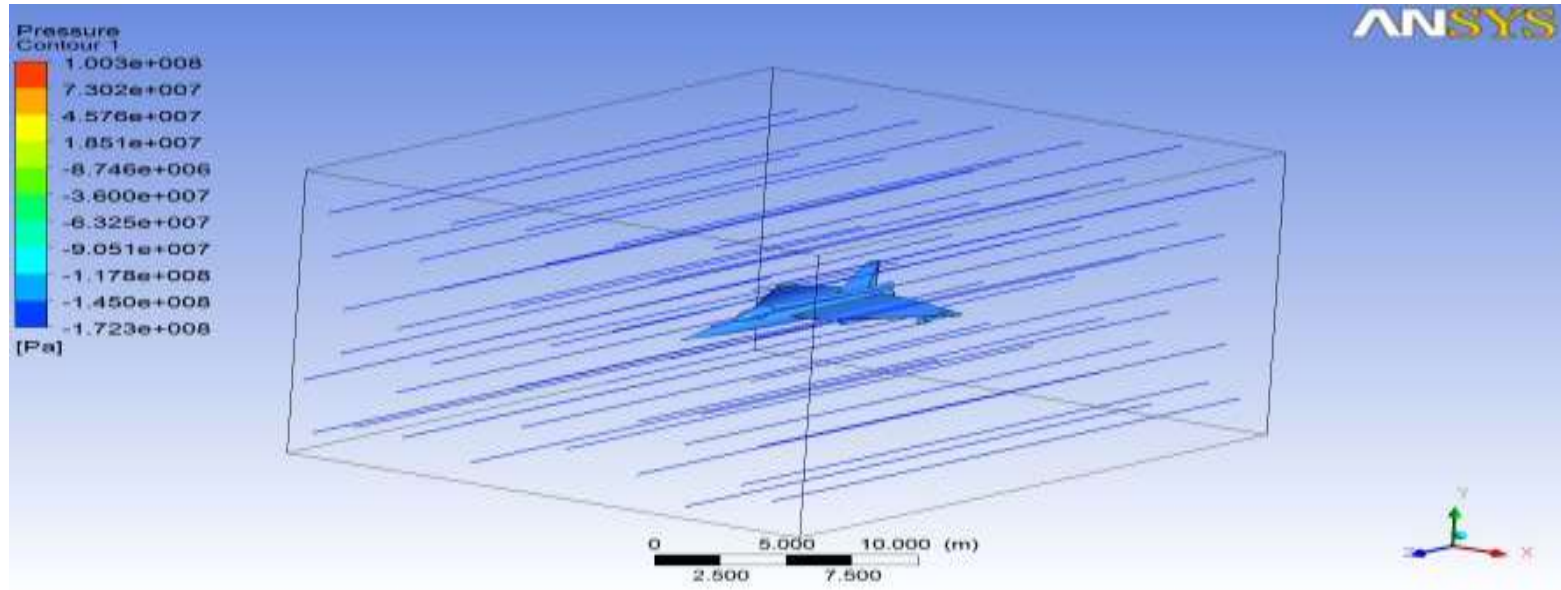

Fig.3.2: Pressure contour at normal degree angle. 


\section{RESIDUAL PLOT:}
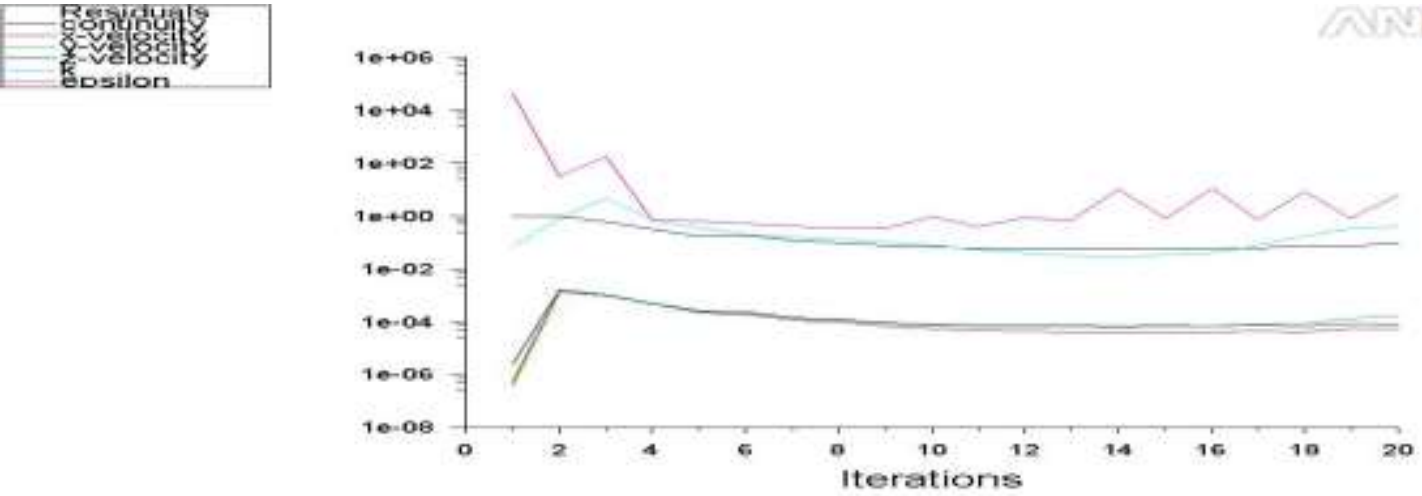

seaied Residuals

ANEYS FLUENT 140 (3d, Sep 25, 2015

Graph I: It shows the iterations of residual results.

\section{Test case II:}

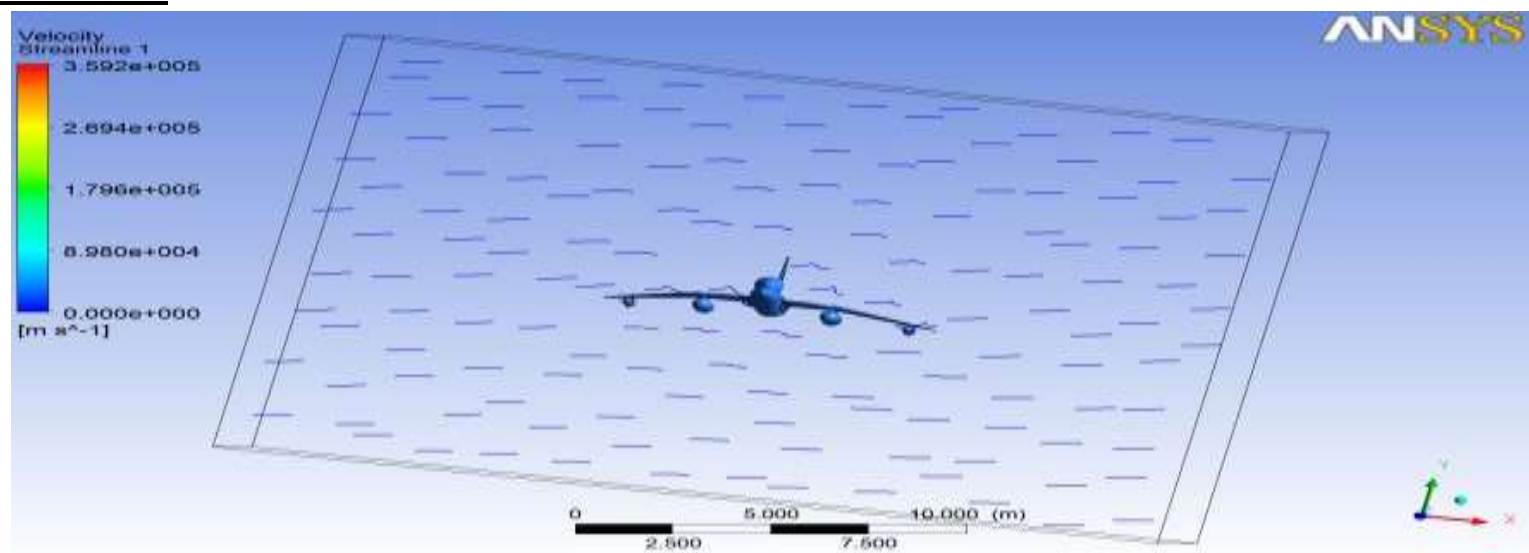

Fig.4.1: Velocity contour at 4 degree angle.

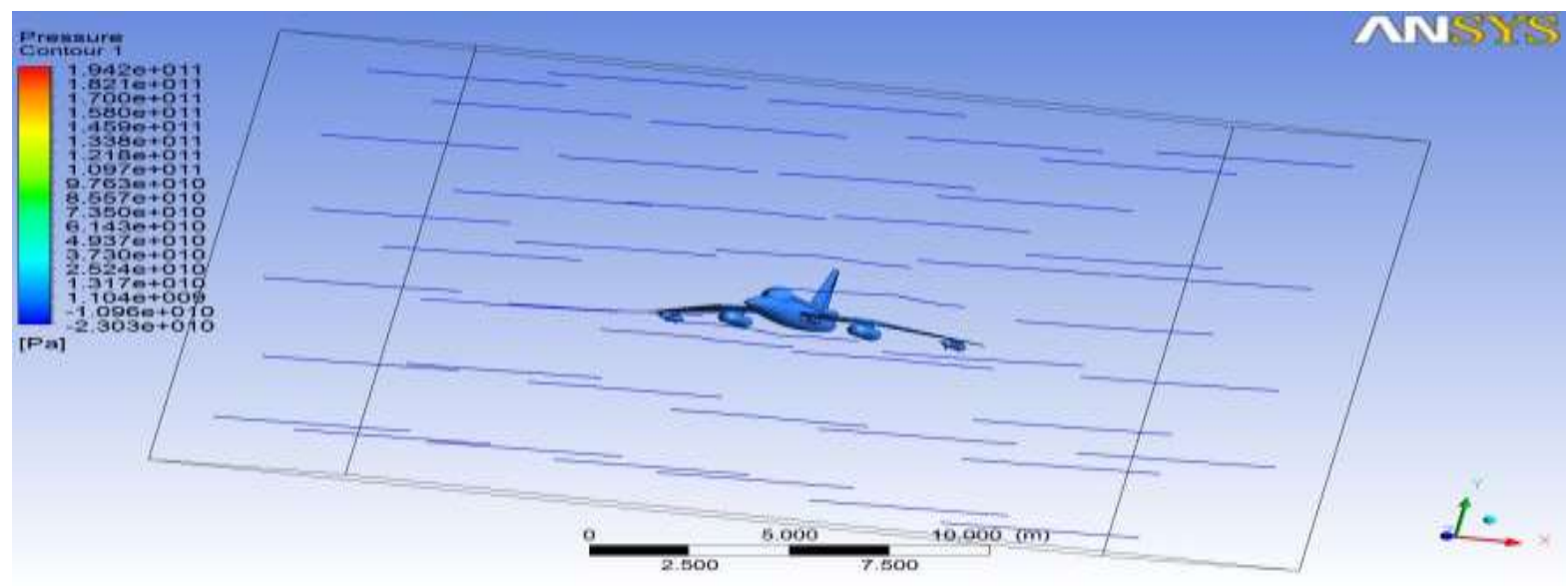

Fig.4.2: Pressure contour at 4 degree angle 

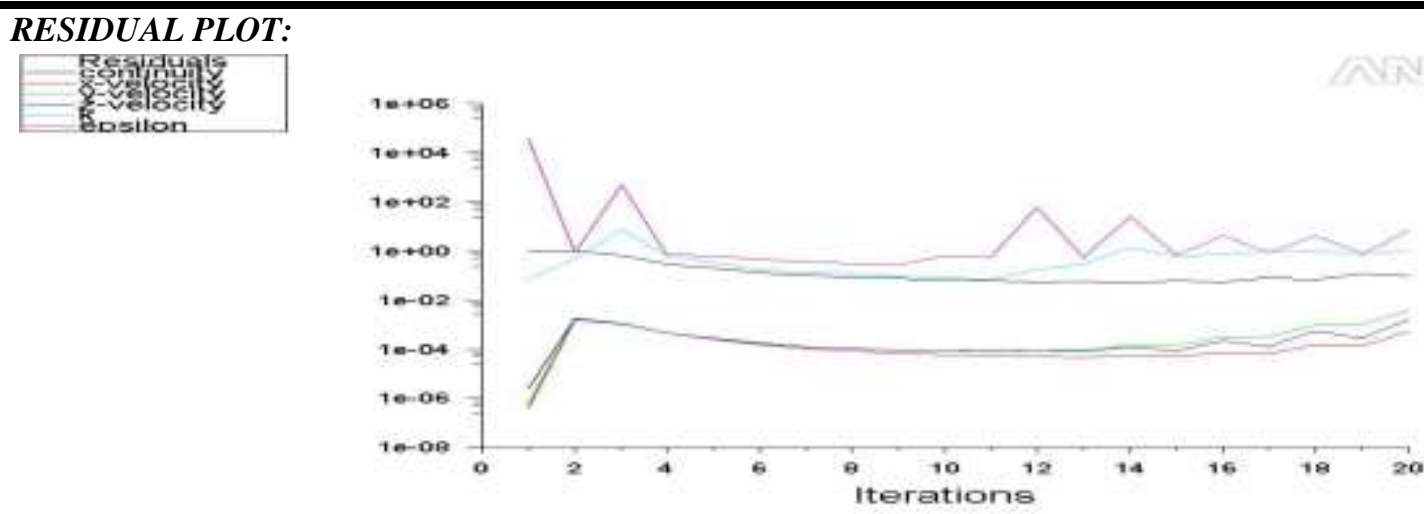

Graph II : It shows iterations of residual results.

$\underline{\text { Test case III: }}$

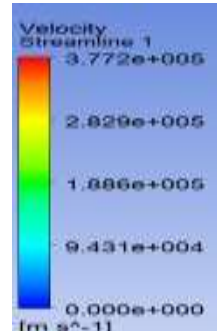
$\operatorname{tm}: 0.000$

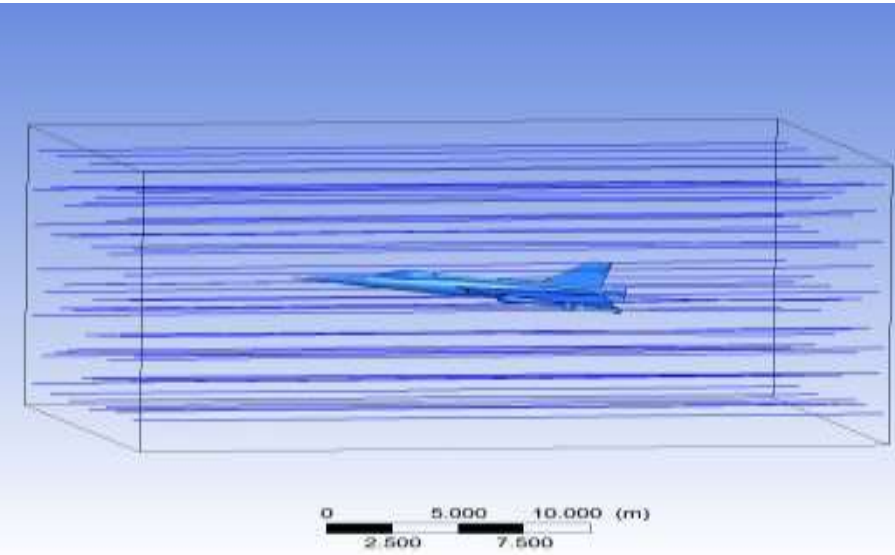

ANSTE

Fig.5.1: Velocity contour at 8 degree angle.

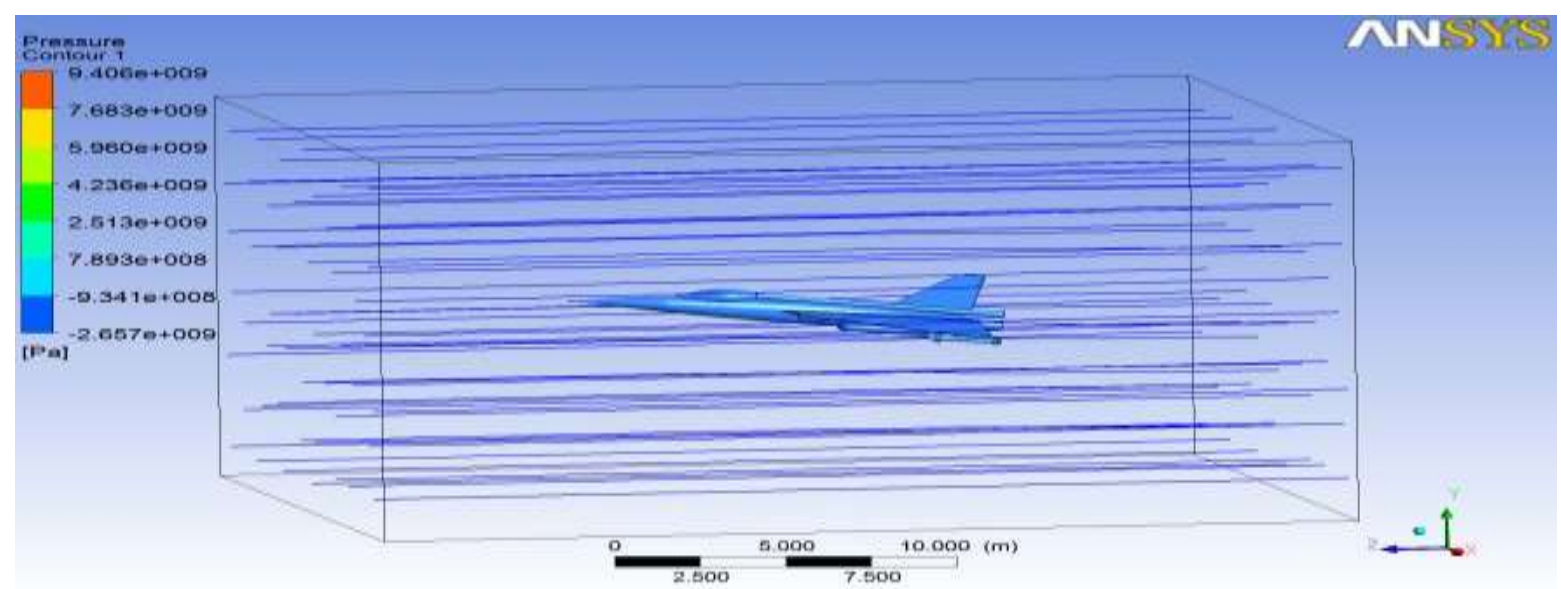

Fig.5.2: Pressure contour at 8 degree angle 

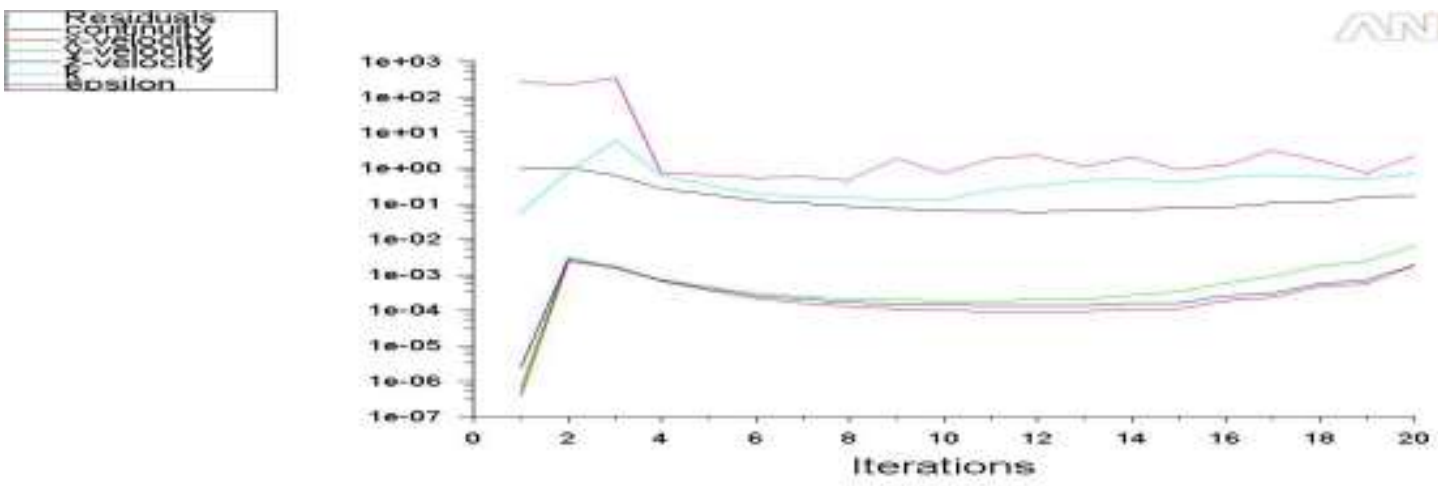

Graph III : It shows iterations of residual results.

The above figures and graphs show the results being drawn in case of pressure distribution, velocity of air and density of the aircraft. During the movement of the aircraft in the stream of air, the velocity flows along the upper and lower surface of the aircraft and also along the leading edge of the aircraft. Further at downstream, the velocity moves along the tailing edge and this velocity is considered to be high i.e. approximately $85 \%$ of the free stream velocity. According to Bernoulli's equation, as velocity increases the pressure decreases and vice versa. Thus a higher pressure is observed at lower surface of the body which is more than the ambient pressure at the upper surface of the aircraft body that is considered to be low. Further by increasing the "angle of attack", the velocity distribution also changes which can be seen in the above figures, shown at 'angle of attack' of 0 degrees, 4 degrees, 8 degrees respectively. From the above figures, it is observed that there is an increase in velocity at the upper surface while a decrease at the lower surface. Also an increase in pressure difference will result in an increase in the lift and is found to be same in case of density also. We can observe from the figures that for each "angle of attack", the flow of velocity and the distribution of pressure vary. The value velocity at the top and bottom surface of aircraft at normal degree of "angle of attack" is relatively small. Hence the value of pressure distribution at this point is also small resulting in a small lifting force. At this point the flow over the wing still remains dominant. On the other hand there is large amount of energy loses during the separation of boundary layer and this energy loss in most applications adversely affects the aerodynamic lift and increase in drag. So an increase in "angle of attack" increases the drag while the reduction in the drag results in energy and fuel consumption. The above figures show mostly the positive pressures than the negative pressures. So an increase in "angle of attack" results an increase in coefficient of lift, affecting the aerodynamic characteristics of the body. At this state, the flow separation tends to act at the bottom edge of the body since the pressure at the bottom edge is less than the pressure at the top edge. The resultant pressure is the sum of the top surface positive pressure and bottom surface negative pressure. However sometimes due to changes in the design conditions, the bottom surface is carrying the positive pressure while the top surface is carrying the negative pressure. The positive pressure at the bottom edge determines the shape and thickness of the airfoil. Large positive values at the bottom edge imply more severe adverse pressure gradients. Thus due the increase in "angle of attack", the pressure increases from the minimum at the top edge to the value at the bottom edge which is considered to be maximum at that point. This total region is considered as the pressure recovery region and this pressure is associated with the boundary layer transitions. Thus finally the flow separation occurs when the pressure is too high at higher "angle of attack".

\section{GRAPHS AND TABLE}

After performing required simulation, the values of Velocity, pressure and density with a different "angle of attack" were observed. After observing the results, the following graphs at each degree are drawn in order show the increase and decrease in the respective values giving an idea about the performance of the aircraft to analyse 
Graph showing the results of the test I:
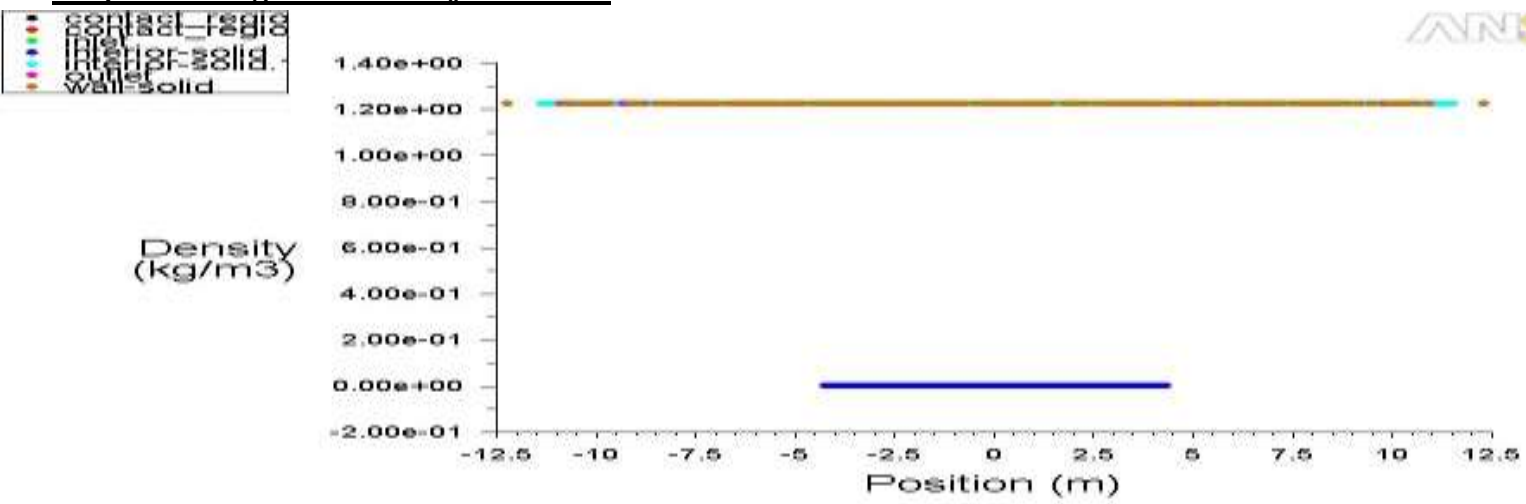

Density

Graph 1.1: Density graph of the aircraft at normal angle

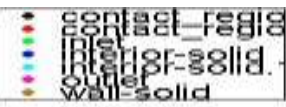

Static Pressure ANSYS FLUENT 14.0 (3d, dp, pbns, sko)

Graph 1.2: Pressure graph at normal angle

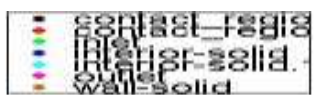

$M=a g n i t$ ist (in/:
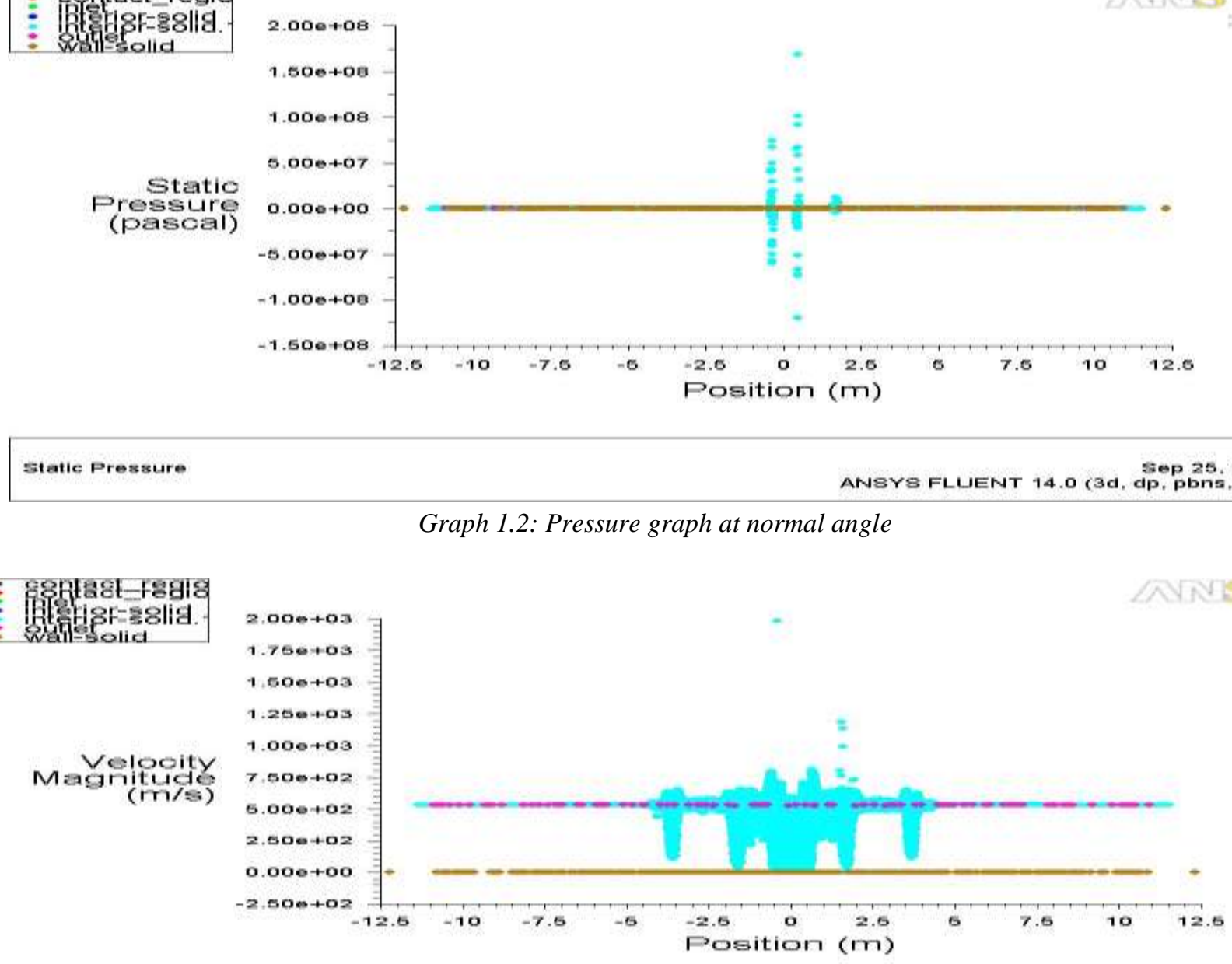
Graphs showing the results of test II:

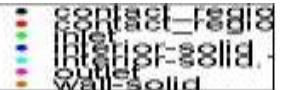

$6.000+03$

$4.000+03$

$3,500+03$

3.00e+os

$2.600+03$

Velooity

( $\mathrm{m} / \mathrm{s}$ )

$2.000+03$

$1.500+03$

$1,000+03$

$6.000+02$

$0,000+00$

$-6.000+02$

$-12.6$

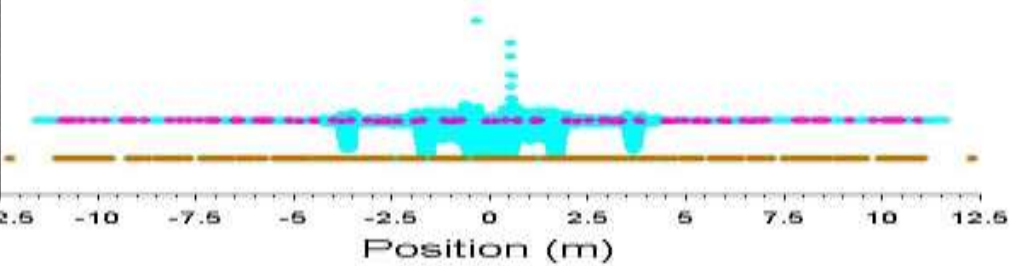

Veloeity Magnitude

ANEVE FLUENT 14,0 (3d, dp, pbris, ske)

Graph 2.1: Velocity magnitude graph of the aircraft at 4 degree angle

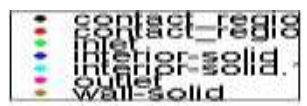

$2,000+11$

$1,75 e+11$

$1,506+11$

$1.25 e+11$

$1,000+11$

Statio

Prossure
(pascal)

$7.500+10$

$5,000+10$

$2.500+10$

$0.00 \mathrm{a}+00$

$-2.500+10$

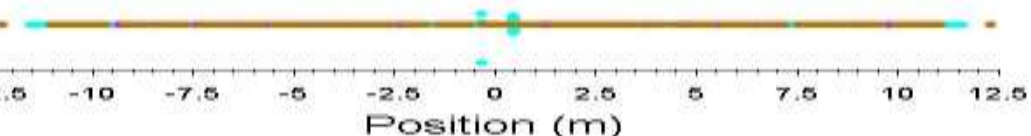

Graph2.2: Pressure graph at 4 degree angle

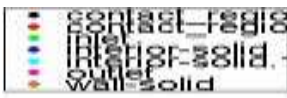

$1,200+00$

1.00e+oo

8.000 .01

Derisity

6.000-01

4.000-01

2.000-01

$0.000+00$

$-2.000-01$

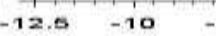

$\begin{array}{ll}-2.5 & 0 \\ \text { position }(m)\end{array}$

Density ANSYS FLUENT 140 (3d 80 P 27,2016

Graph2.3: Density graph at 4 degree angle 
Graph showing the results of test III:
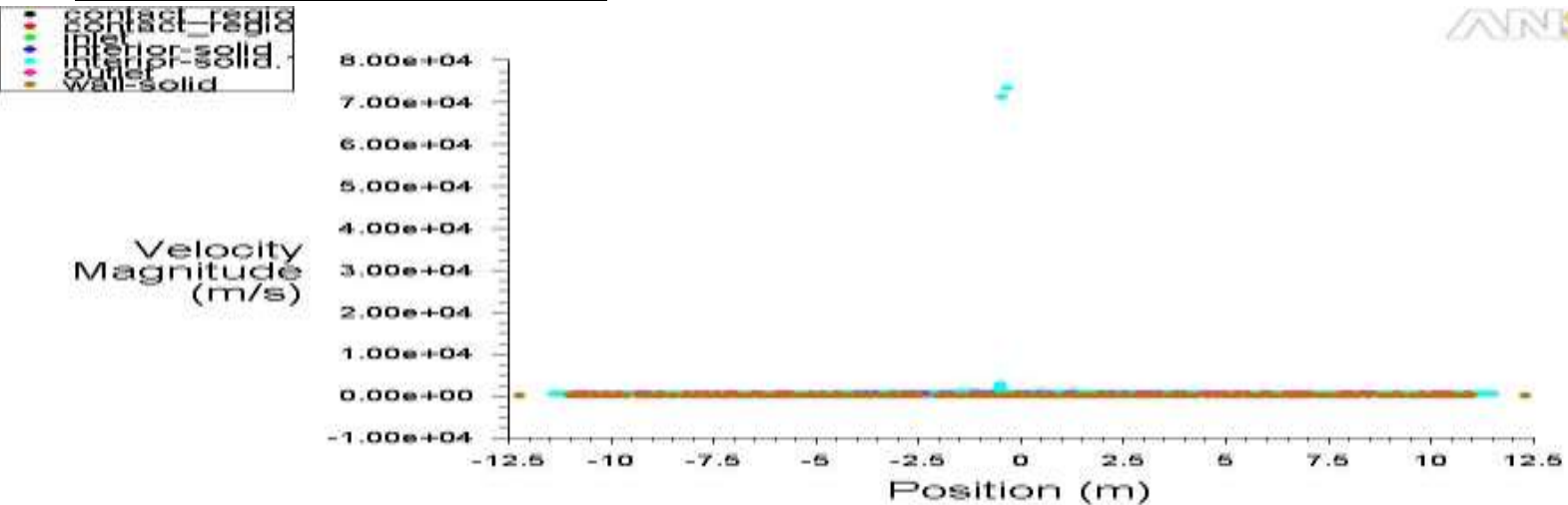

Graph 3.1: Velocity magnitude graph at 8 degree angle

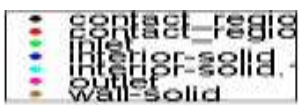

$$
\begin{aligned}
& \text { Solid } \\
& \text { Sressure } \\
& \text { (pasoai) }
\end{aligned}
$$

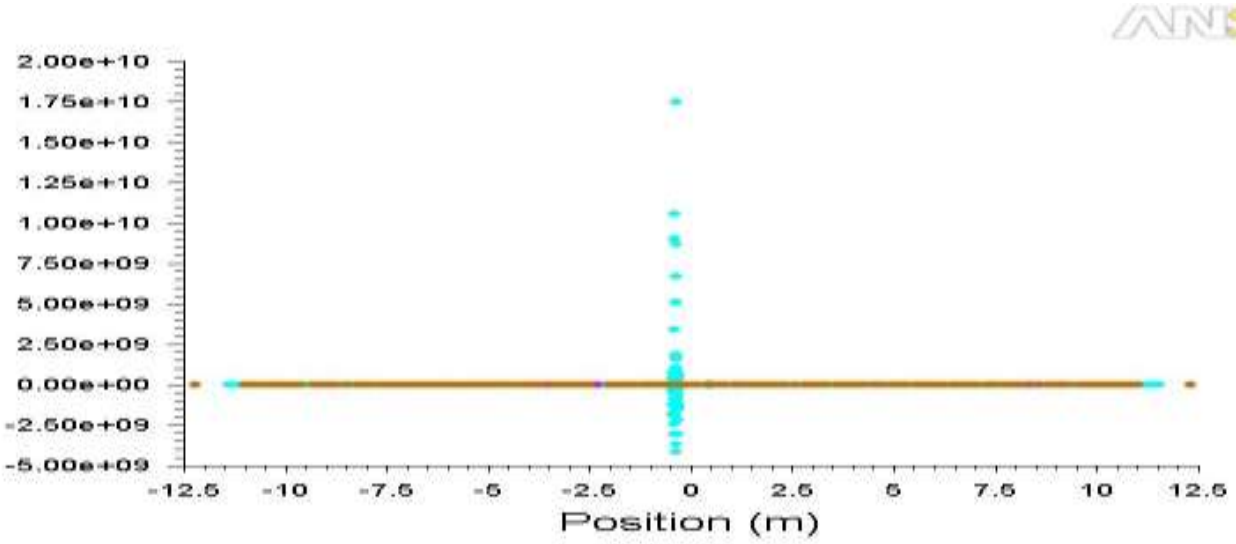

Graph3.2: Pressure graph at 8 degree angle

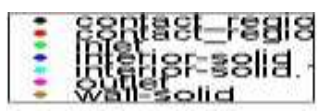

(ken/mity

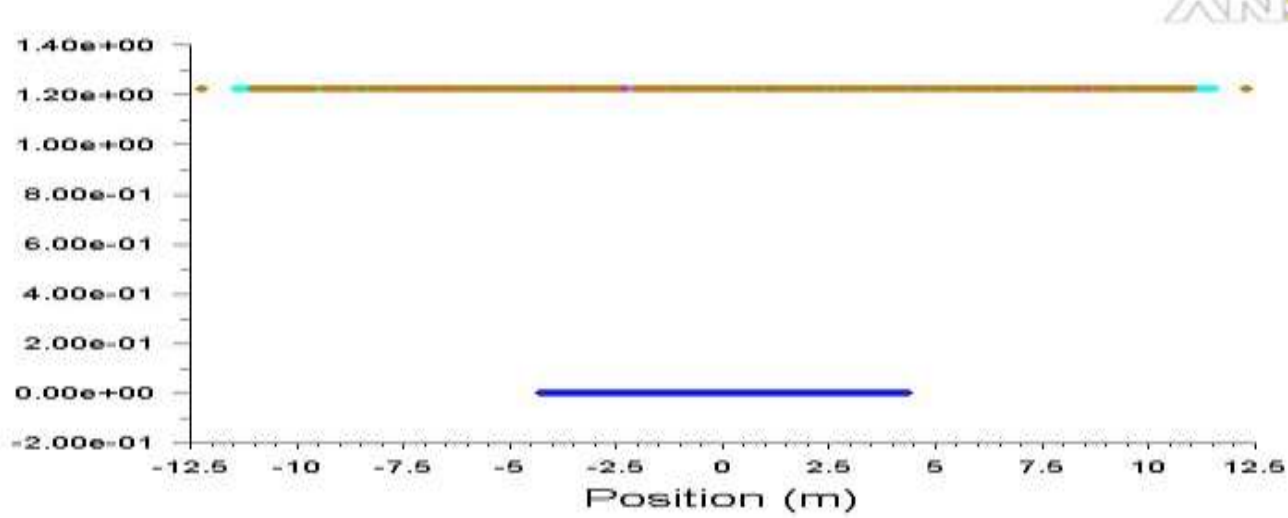

Graph 3.3: Density graph at 8 degree angle 


\section{CONCLUSSION}

This study shows the following conclusion:

After the analysis of the simulated flow over the aircraft on different properties considered, the following conclusions were drawn

- As "angle of attack" increases, the velocity increases and centre of pressure difference moves forward. If the angle of attack decreases the pressure difference moves rearward i.e. towards the bottom edge. .I

- For a normal degree of "angle of attack" with a speed of $533 \mathrm{~m} / \mathrm{s}$, it is observed that the maximum velocity is $2.405 \mathrm{e}+004 \mathrm{~m} / \mathrm{s}$ and total pressure acting is $1.003 \mathrm{e}+008 \mathrm{pa}$.

- At 4degree of "angle of attack" with speed of 533 $\mathrm{m} / \mathrm{s}$, it is observed that maximum velocity is $3.592 \mathrm{e}+005 \mathrm{~m} / \mathrm{s}$, and total pressure is about $1.942 \mathrm{e}+011 \mathrm{pa}$.

- At 8degree of “angle of attack' with speed of $533 \mathrm{~m} / \mathrm{s}$, it is observed that maximum velocity is $3.772 \mathrm{e}+005 \mathrm{~m} / \mathrm{s}$ and total pressure is about $9.406 \mathrm{e}+009 \mathrm{pa}$.

- Finally from the values of the result it can concluded that the drag and lift coefficient of the aircraft depends on the airfoil shape and also on the velocity distribution.

\section{REFERENCES}

[1] Antony Jameson and Massimiliano Fatica, "Using Computational Fluid Dynamics for Aerodynamics" Stanford University.

[2] Beechook.A, Wang. J, "Aerodynamic Analysis of Variable Cant Angle Winglets for Improved Aircraft Performance", Department of Aerospace, Electrical \& Electronic Engineering, Coventry University Coventry, United Kingdom.

[3] Christopher L. Rumsey, Susan X. Ying et al, “A CFD Prediction of High Lift : review of present CFD capability", Elsevier, Progress in Aerospace Sciences, volume 38, issue 2, February 2002.

[4] Christopher L.Rumsey, "Summary of the First AIAA CFD High Lift Prediction Workshop" (invited), NASA Langley Research Center, Hampton VA M. Long† University of Wyoming.

[5] Gary B. "Computational Fluid Dynamics Analysis Success Stories of X-plane Design to Flight Test",
Cosentino NASA Dryden Flight Research Center Edwards, California.

[6] Le Chuiton.F, Alascio.A.D, Barakos.G, et al, "Computation of the Helicopter Fuselage Wake with the SST, SAS, DES and XLES Models", Volume97, pp 117-124, Springer eBook, January 24, 2008.

[7] Mueller, T. J., (editor), "Aerodynamic characteristics of low aspect ratio wings at low Reynolds numbers" presented at the conference on fixed, flapping and rotary wing vehicles at very low Reynolds numbers, Notre Dame.

[8] Nathan Logsdon, Dr. Gary Solbrekken, "Procedure For Numerically Analyzing Airfoils And Wing Sections" Volume 2, Issue2 doc toral diss", University of Missouri Columbia, December 2006.

[9] Ryan Babigian and Shigeo Hayashibara (2009): "Computational study of the Vortex Wake Generated by a Three-Dimensional Wing with Dihedral, Taper and Sweep", 27th AIAA Applied Aerodynamics, Conference 22-25 June 2009.

[10] Singh.K.P, Mathur J.S, Ashok.V, and Debasis Chakraborty, "Computational Fluid Dynamics in Aerospace Industry in India", Volume 60, Number: 6, Defence Science Journal, 2010.

[11] Shivasharanayya Hiremath1, Anandkumar. S. Malipatild,"CFD Simulations of Aircraft Body with Different Angle of Attack and Velocity",Department of Thermal Power Engineering, VTU PG center, Gulbarga, India.

[12] Tejas - India's Light Combat Aircraft

[13] Takashi Misaka, Frank Holzapfel and Thomas Gerz, "Large Eddy Simulation of Wake Vortex Evolution from Roll-Up to Vortex Decay" on $49^{\text {th }}$ AIAA Aerospace Science meeting including the New Horizons forum and Aerospace Exposition (AIAA 2011 1003), 4-7 January 2011, Orlando, Florida.

[14] Wortmann.F.X, "The Quest for High Lift at Low Reynolds Number", AIAA Paper 74-1018, MITCambridge, Sept. 1974.

[15] https://en.wikipedia.org/wiki/Angle_of_attack 